\title{
Rectifications of $\mathrm{A}_{\infty}$-algebras ${ }^{1}$
}

\author{
M. J. Jiménez and P. Real
}

Dpto. Matematica aplicada I, Universidad de Sevilla, Spain

\begin{abstract}
In this paper, in the setting of connected DG-modules, we prove that, for any $A_{\infty^{-}}$ algebra $\left(M, m_{1}, m_{2}, m_{3}, \ldots\right)$, there is a chain contraction from a DG-algebra onto the DG-module $M$ such that the $A_{\infty}$-algebra structure induced by perturbation theory on $M$ is the original one. In fact, the mentioned DG-algebra is a rectification of the $A_{\infty}$-algebra in the sense of Boardman and Vogt [BV73]. Appropiated dual results are given for $A_{\infty}$-coalgebras.
\end{abstract}

Key Words: $A_{\infty}$-algebra, $A_{\infty}$-coalgebra, contraction, tilde bar construction, tilde cobar construction

MSC: 18E30 18G35 16E45

\section{Introduction}

One classical result claims that each $W P$-algebra (strongly homotopy $P$-algebra) can be replaced by a strict $P$-algebra of the same homotopy type. More concretely, for each $W P$-algebra, $X$, there exists a strict $P$-algebra, $M X$, together with a strong deformation retraction $(M X, X, r: M X \rightarrow X, i: X \rightarrow M X)$ in which $i$ is a homomorphism of $W P$ algebras [BV73]. Then, $M X$ is called the rectification of $X$.

It seems to be a commonly accepted fact that the analogous statement holds also for $A_{\infty}$-algebras, that is, each $A_{\infty}$-algebra can be replaced by an associative DG-algebra of the same homotopy type. We have not been able, however, to find a proof of this statement in the literature.

The aim of this paper is to construct such a rectification of an $A_{\infty}$-algebra explicitly.

It is known that a chain contraction, which is a special type of chain homotopy equivalence, from a DGA-algebra $A$ onto a DG-module $M$ provides an $A_{\infty}$-algebra structure on $M$ via the tensor trick [Gug77, GS86, GLS91]. In fact, the first transference of an $A_{\infty^{-}}$ algebra structure, in this sense, was made by Kadeishvili in [Kad80] for the case $M=H(A)$.

The main result in this paper can be seen as an inverse procedure of that one. In other words, we assert that, working in the context of connected DG-modules, for any

\footnotetext{
${ }^{1}$ This work was partially supported by the PAICYT research project FQM-296 from Junta de Andalucía (Spain).

Address correspondence to Maria Jose Jimenez, Depto. Matematica Aplicada I, E.T.S.I. Informatica, Universidad de Sevilla, Avda. Reina Mercedes, S.N. 41012 Sevilla (Spain)
} 
$A_{\infty}$-algebra $\left(M, m_{1}, m_{2}, m_{3}, \ldots\right)$, there exists a DG-algebra, $A_{M}$, and a chain contraction from $A_{M}$ to $M$, such that the application of the tensor trick to this contraction yields the original $A_{\infty}$-algebra structure on $M$, defined by the family of morphisms $\left\{m_{i}\right\}$. In the dual case of $A_{\infty}$-coalgebras, our framework is the one of simply connected DG-modules.

In order to prove this result, we construct, by means of homological perturbation theory [HK91, GL89, GLS91], a chain contraction from the reduced cobar construction of the tilde bar construction of $M, \bar{\Omega} \widetilde{B}(M)$, to the DG-module $M$. This contraction represents a generalization (in the setting of $A_{\infty}$-algebras) of the result given by Munkholm in [Mun74] by which, for a DGA-algebra $A$, a contraction (called trivialized extension) from the DGAalgebra $\bar{\Omega} \bar{B}(A)$ to $A$ is described.

This paper is organized as follows: in the next section we recall the preliminaries needed and state the notation used throughout the paper; the third section is devoted to our description of a family of morphisms that provide an $A_{\infty}-$ (co)algebra structure via the tensor trick; in the forth section, we establish the main result of the paper by which any $A_{\infty^{-}}$ (co)algebra can be seen as a chain contraction from a (simply) connected DG-(co)algebra onto a DG-module.

\section{Notations and preliminaries}

Although relevant notions of homological algebra are recalled here, most of common concepts are not explicitly given. They might be consulted, for instance, in [CE56] and [Mac95].

Take a commutative ground ring, $\Lambda$, with unit. A differential graded module or DGmodule, $(M, d)$, is a module $M$, graded on the non-negative integers, $M=\bigoplus_{n>0} M_{n}$, endowed with a morphism of graded modules $d$ of degree -1 such that, $d^{2}=0$. A DGmodule $M$ is called connected if $M_{0}=\Lambda$ and simply connected if it is connected and $M_{1}=0$. Given a connected DG-module, $M$, the reduced module $\bar{M}$ is such that $\bar{M}_{n}=M_{n}$ for $n>1$ and $\bar{M}_{0}=0$. In this paper, we shall always refer to connected DG-modules in the context of $A_{\infty}$-algebras and simply connected DG-modules when dealing with $A_{\infty}$-coalgebras.

We will denote the module $M \otimes \stackrel{n}{n} \otimes M$ by $M^{\otimes n}$, with $M^{\otimes 0}=\Lambda$; we will use the notation $f^{\otimes n}$ for the morphism

$$
f \otimes \cdots \cdot \otimes f: M^{\otimes n} \rightarrow N^{\otimes n} .
$$

We will respect the Koszul convention for signs.

On the other hand, if $f: M^{\otimes i} \rightarrow N$ is a DG-module morphism and $n$ is a non-negative integer, we will denote by $f^{[n]}: M^{\otimes n} \rightarrow N^{\otimes n-i+1}$ the morphism

$$
f^{[n]}=\sum_{j=0}^{n-i} 1^{\otimes j} \otimes f \otimes 1^{\otimes n-i-j} .
$$

The morphism $f^{[]}: \bigoplus_{j \geq i} M^{\otimes j} \rightarrow \bigoplus_{k \geq 1} N^{\otimes k}$ will be the one such that $\left.f^{[]}\right|_{M^{\otimes n}}=f^{[n]}$.

If $\left(M, d_{M}\right)$ is a DG-module, the suspension of $M$ is defined as the DG-module $\left(s M, d_{s M}\right)$, where $(s M)_{n}=M_{n-1}$ and $d_{s M}=-d_{M}$. The desuspension of $M$ is given by $\left(s^{-1} M\right)_{n}=M_{n+1}$ 
and differential $-d_{M}$ too. We will denote by $\uparrow$ and $\downarrow$ the suspension and desuspension morphisms, which shift the degree by +1 and -1 , respectively. A given morphism of graded modules of degree $k, f: M \rightarrow N$, induces $s f: s M \rightarrow s N$, given by $s f=(-1)^{k} \uparrow f \downarrow$. In fact, $d_{s M}=s d_{M}=-\uparrow d_{M} \downarrow$.

A $D G$-algebra, $\left(A, d_{A}, \mu_{A}\right)$, is a DG-module endowed with an associative product, $\mu_{A}$, compatible with the differential $d_{A}$ and which has a unit $\eta_{A}: \Lambda \rightarrow A$, that is, $\mu_{A}\left(\eta_{A} \otimes 1\right)=$ $\mu_{A}\left(1 \otimes \eta_{A}\right)=1$. Sometimes we will use the notation $*_{A}$ for the product on $A$. If there is no confusion, subscripts will be omitted. A $D G$-coalgebra $\left(C, d_{C}, \Delta_{C}\right)$ is a DG-module provided with a compatible coproduct and counit $\xi_{C}: C \rightarrow \Lambda\left(\right.$ so, $\left.\left(\xi_{C} \otimes 1\right) \Delta_{C}=\left(1 \otimes \xi_{C}\right) \Delta_{C}=1\right)$.

Given a DG-module $(M, d)$, the tensor module of $M$ is denoted by $T(M)$ and is constructed in the following way:

$$
T(M)=\bigoplus_{n \geq 0} M^{\otimes n}
$$

The tensor graduation of $T(M),||_{t}$, is given by:

$$
\left|a_{1} \otimes \cdots \otimes a_{n}\right|_{t}=\sum_{i=1}^{n}\left|a_{i}\right| .
$$

The differential structure in $T(M)$ is provided by the tensor differential, $d_{t}$, which is the morphism $d_{M}^{[]}$.

A product, $\mu$, and a coproduct, $\Delta$, can be naturally defined on $T(M)$, as follows:

- $\mu\left(\left(a_{1} \otimes \cdots \otimes a_{n}\right) \otimes\left(a_{n+1} \otimes \cdots \otimes a_{n+p}\right)\right)=a_{1} \otimes \cdots \otimes a_{n+p} ;$

- $\Delta\left(a_{1} \otimes \cdots \otimes a_{n}\right)=\sum_{i=0}^{n}\left(a_{1} \otimes \cdots \otimes a_{i}\right) \otimes\left(a_{i+1} \otimes \cdots \otimes a_{n}\right)$.

Therefore, $T(M)$ acquires both structures of DG-algebra (denoted by $T^{a}(M)$ ) and DGcoalgebra $\left(T^{c}(M)\right)$, though they are not compatible to each other (that is, $(T(M), \mu, \Delta)$ is not a Hopf algebra).

Every morphism of DG-modules $f: M \rightarrow N$ induces another one $T(f): T(M) \rightarrow T(N)$, such that $\left.T(f)\right|_{M \otimes n}=f^{\otimes n}$.

The reduced bar construction of a connected DG-algebra $A, \bar{B}(A)$, is a DG-coalgebra whose module structure is given by

$$
T(s \bar{A})=\bigoplus_{n \geq 0}(s \bar{A})^{\otimes n} .
$$

A typical element of $\bar{B}(A)$ is denoted by $\bar{a}=\left[a_{1}|\cdots| a_{n}\right]$ and []$=1 \in \Lambda$. The degree of $a$ is given by the sum of the tensor degree $\left|a_{1}\right|+\cdots+\left|a_{n}\right|$ and the simplicial degree $n$, which appeals to the number of components or length of the element (also referred to as simplicial dimension).

The total differential $d_{\bar{B}}$ is given by the sum of the tensor differential, $d_{t}$ (which is the natural one on the tensor product) and the simplicial differential, $d_{s}$ (that depends on the product on $A$ ):

$$
d_{t}=-\left(\uparrow d_{A} \downarrow\right)^{[]} ; \quad d_{s}=\left(\uparrow \mu_{A} \downarrow^{\otimes 2}\right)^{[]} .
$$


The coproduct $\Delta_{\bar{B}}: \bar{B}(A) \rightarrow \bar{B}(A) \otimes \bar{B}(A)$ is the natural one on the tensor module.

Given a simply connected DG-coalgebra $C$, the reduced cobar construction, $\bar{\Omega}(C)$, is a DG-algebra whose underlying module is

$$
T\left(s^{-1} \bar{C}\right)=\bigoplus_{n \geq 0}\left(s^{-1} \bar{C}\right)^{\otimes n} .
$$

A typical element of $\bar{\Omega}(C)$ will be written $\bar{c}=\left\langle c_{1}|\cdots| c_{n}\right\rangle$, being \langle\rangle$=1 \in \Lambda$. The total degree of $\bar{c}$ is $|\bar{c}|_{\bar{\Omega}}=\left|c_{1}\right|+\cdots\left|c_{n}\right|-n$. We will refer to the length of the element, $n$, as the cosimplicial degree (or dimension).

The total differential $d_{\bar{\Omega}}$ is given by the sum of the tensor differential and the cosimplicial differential $d_{c o s}=\left(\downarrow^{\otimes 2} \Delta_{C} \uparrow\right)^{[]}$. The product on $\bar{\Omega}(C)$ is the natural one on the underlying module.

In the context of homological perturbation theory, the main input data are chain contractions (or simply, contractions) [EM53, GS86, LS87, GL89, HK91]: a contraction $c:\{N, M, f, g, \phi\}$ from a DG-module $N$ to a DG-module $M$, consists in a particular homotopy equivalence determined by three morphisms $f, g$ and $\phi$; being $f: N_{\star} \rightarrow M_{\star}$ (projection) and $g: M_{\star} \rightarrow N_{\star}$ (inclusion) two DG-module morphisms and $\phi: N_{\star} \rightarrow N_{\star+1}$ a homotopy operator, that is, $f g=1_{M}$, and $\phi d_{N}+d_{N} \phi+g f=1_{N}$. Moreover, these data are also required to satisfy:

$$
f \phi=0, \quad \phi g=0, \quad \phi \phi=0 .
$$

Notice that the homology of both DG-modules are isomorphic. We will also use the notation $(f, g, \phi): N \Rightarrow M$ or simply $N \stackrel{c}{\Rightarrow} M$ when confusion cannot arise.

Given a DG-module contraction,

$$
c:\{N, M, f, g, \phi\}
$$

we can establish the following ones [GL89, GLS91]:

- The suspension contraction of $c, s c$, which consists of the suspended DG-modules and the induced morphisms:

$$
s c:\{s N, s M, s f, s g, s \phi\},
$$

being $s f=\uparrow f \downarrow, s g=\uparrow g \downarrow$ and $s \phi=-\uparrow \phi \downarrow$, which are briefly expressed by $f, g$ and $-\phi$.

- The tensor module contraction, $T(c)$, between the tensor modules of $N$ and $M$ :

$$
T(c):\{T(N), T(M), T(f), T(g), T(\phi)\},
$$

where

$$
\left.T(\phi)\right|_{N \otimes n}=\phi^{[\otimes n]}=\sum_{i=0}^{n-1} 1^{\otimes i} \otimes \phi \otimes(g f)^{\otimes n-i-1} .
$$


Now, we recall the concept of perturbation datum. Let $N$ be a graded module and let $f: N \rightarrow N$ be a morphism of graded modules. The morphism $f$ is defined to be pointwise nilpotent whenever for all $x \in N, x \neq 0$, there exists a positive integer $n$ such that $f^{n}(x)=0$. A perturbation of a $D G$-module $N$ consists in a morphism of graded modules $\delta: N \rightarrow N$ of degree -1 , such that $\left(d_{N}+\delta\right)^{2}=0$. A perturbation datum of the contraction $c:\{N, M, f, g, \phi\}$ is a perturbation $\delta$ of the DG-module $N$ satisfying that the composition $\phi \delta$ is pointwise nilpotent.

The main tool when dealing with contractions is the Basic Perturbation Lemma [Bro67, Gug72, LS87], which is an algorithm whose input is a contraction of DG-modules $c:\{N, M, f, g, \phi\}$ and a perturbation datum $\delta$ of $c$ and whose output is a new contraction $c_{\delta}:\left\{\left(N, d_{N}+\delta\right),\left(M, d_{M}+d_{\delta}\right), f_{\delta}, g_{\delta}, \phi_{\delta}\right\}$ defined by the formulas

$$
d_{\delta}=f \delta \Sigma_{c}^{\delta} g ; \quad f_{\delta}=f\left(1-\delta \Sigma_{c}^{\delta} \phi\right) ; \quad g_{\delta}=\Sigma_{c}^{\delta} g ; \quad \phi_{\delta}=\Sigma_{c}^{\delta} \phi ;
$$

where $\Sigma_{c}^{\delta}=\sum_{i \geq 0}(-1)^{i}(\phi \delta)^{i}$.

The pointwise nilpotency of the composition $\phi \delta$ guarantees that the sums are finite for each particular element.

\section{From contractions to $A_{\infty}$-structures}

We find the origin of $A_{\infty}-(\mathrm{co})$ algebras in [Sta63], where Stasheff set the concept of strongly homotopy associativity in the search of a homotopy invariant that plays the role of associativity.

We recall here the definition of $A_{\infty}$-algebra (resp. $A_{\infty}$-coalgebra) [Kad80, Pro84]. An $A_{\infty}$-algebra (respectively, $A_{\infty}$-coalgebra), is a DG-module $\left(M, m_{1}\right)$ (resp., $\left.\left(M, \Delta_{1}\right)\right)$ endowed with a family of morphisms of graded modules

$$
m_{i}: M^{\otimes i} \rightarrow M\left(\text { resp., } \Delta_{i}: M \rightarrow M^{\otimes i}\right)
$$

of degree $i-2$ such that, for $i \geq 1$,

$$
\begin{gathered}
\sum_{n=1}^{i} \sum_{k=0}^{i-n}(-1)^{n+k+n k} m_{i-n+1}\left(1^{\otimes k} \otimes m_{n} \otimes 1^{\otimes i-n-k}\right)=0, \\
\text { (resp., } \left.\sum_{n=1}^{i} \sum_{k=0}^{i-n}(-1)^{n+k+n k}\left(1^{\otimes i-n-k} \otimes \Delta_{n} \otimes 1^{\otimes k}\right) \Delta_{i-n+1}=0\right) .
\end{gathered}
$$

Starting from a contraction between a connected DG-algebra $A$ and a DG-module $M$, the application of the tensor trick [Gug77, GS86, GLS91] provides a way of constructing a family of morphisms that makes the module inherit an $A_{\infty}$-algebra structure. Using this technique, in the following theorem, we will express these morphisms with regard to the component morphisms of the initial contraction. We draft a proof of the theorem with the only purpose of showing the tools used in the context of homological perturbation theory. 
Theorem 3.1. [Kad80, GLS91] Let $\left(A, d_{A}, \mu\right)$ and $\left(M, d_{M}\right)$ be a connected DG-algebra and a $D G$-module, respectively and $c:\{A, M, f, g, \phi\}$ a contraction between them. Then the $D G$-module $M$ is endowed with an $A_{\infty}$-algebra structure by the morphisms

$$
\begin{aligned}
& m_{1}=-d_{M} \\
& m_{n}=(-1)^{n+1} f \mu^{(1)} \phi^{[\otimes 2]} \mu^{(2)} \cdots \phi^{[\otimes n-1]} \mu^{(n-1)} g^{\otimes n}, \quad n \geq 2
\end{aligned}
$$

where

$$
\mu^{(k)}=\sum_{i=0}^{k-1}(-1)^{i+1} 1^{\otimes i} \otimes \mu_{A} \otimes 1^{\otimes k-i-1} .
$$

Proof.

Starting from $c:\{A, M, f, g, \phi\}$, we can construct the contraction

$$
T(s c):\left\{T^{c}(s \bar{A}), T^{c}(s \bar{M}), T f, T g, T(-\phi)\right\} .
$$

Now, in order to get the differential of the bar construction on the initial DG-module, we consider the simplicial differential as a perturbation datum of this contraction. We can easily check the pointwise nilpotency of $T(-\phi) d_{s}$ since $T(-\phi)$ does not affect to the simplicial dimension of the element, while $d_{s}$ decreases this amount by one and so will be zero after a finite number of steps. Then, by applying the Basic Perturbation Lemma, a new contraction is obtained,

$$
(\widetilde{f}, \widetilde{g}, \widetilde{\phi}): \bar{B}(A) \Rightarrow\left(T^{c}(s \bar{M}), \widetilde{d}\right),
$$

where $\left(T^{c}(s \bar{M}), \widetilde{d}\right)$ is called the tilde bar construction of $M$ [Sta63], denoted by $\widetilde{B}(M)$ and the formula obtained for the perturbed differential is

$$
\widetilde{d}=d_{t}+\sum_{i \geq 0}(-1)^{i} T f d_{s}\left(T(-\phi) d_{s}\right)^{i} T g .
$$

We call

$$
\widetilde{d}_{1}=d_{t}, \quad \widetilde{d}_{i}=(-1)^{i} T f d_{s}\left(T(-\phi) d_{s}\right)^{i-2} T g \quad \text { for } i \geq 2 .
$$

Let us consider the induced morphisms $m_{n}: M^{\otimes n} \rightarrow M$ of degree $n-2$, with the following formulas:

$$
m_{n}=(-1)^{[n / 2]} \downarrow\left(\left.\widetilde{d}_{n}\right|_{(s M)}{ }^{\otimes n}\right) \uparrow^{\otimes n},
$$

where the brackets refer to the integer part. Then, it is easy to check that

$$
m_{n}=(-1)^{n+1} f \mu^{(1)} \phi^{[\otimes 2]} \mu^{(2)} \cdots \phi^{[\otimes n-1]} \mu^{(n-1)} g^{\otimes n} .
$$

Finally, the fact that $\widetilde{d}^{2}=0$ can be translated into relations between the morphisms $m_{n}$ which are, actually, the ones recalled in the formula 1.

An analogous theorem can be established for the case of an $A_{\infty}$-coalgebra structure, whose proof is completely dual to the one given above. 
Theorem 3.2. [Kad80, GLS91] Let $\left(C, d_{C}, \Delta\right)$ and $\left(M, d_{M}\right)$ be a simply connected $D G$ coalgebra and a $D G$-module, respectively, and $c:\{C, M, f, g, \phi\}$ a contraction between them. Then the $D G$-module $M$ is endowed with an $A_{\infty}$-coalgebra structure by the morphisms

$$
\begin{aligned}
& \Delta_{1}=-d_{M} \\
& \Delta_{n}=(-1)^{[n / 2]+n+1} f^{\otimes n} \Delta^{(n)} \phi^{[\otimes n-1]} \Delta^{(n-1)} \cdots \phi^{[\otimes 2]} \Delta^{(2)} g, \quad n \geq 2
\end{aligned}
$$

where

$$
\Delta^{(k)}=\sum_{i=0}^{k-2}(-1)^{i} 1^{\otimes i} \otimes \Delta_{C} \otimes 1^{\otimes k-i-2} .
$$

In this case, the simple connection of the DG-coalgebra guarantees that the formulas implied in the contraction $\bar{\Omega}(C) \Rightarrow \widetilde{\Omega}(M)$, obtained by the Basic Perturbation Lemma, are finite.

Now we are concerned about the inverse process: given an $A_{\infty}-$ (co)algebra, finding a suitable contraction that generates, in the sense given in this section, such a structure.

\section{From $A_{\infty}$-structures to contractions}

In this section, we explicitly construct rectification of an $A_{\infty}$-algebra. As a result, we establish that such a structure can be estructurely represented as a contraction from a DG-algebra onto a DG-module. Recall that by "DG-module" we shall mean a connected DG-module.

Munkholm obtained in [Mun74] a contraction between the reduced cobar construction of the reduced bar construction of a DGA-algebra $A, \bar{\Omega} \bar{B} A$, and the DGA-algebra itself.

$$
c_{A}:\left\{\bar{\Omega} \bar{B} A, A, \alpha_{A}, \rho_{A}, h_{A}\right\} .
$$

The same contraction, can be obtained by means of perturbation theory, for the connected case: starting from a "basic" contraction

$$
\left\{\bar{\Omega} T^{c}(s \bar{A}), A, \alpha, \rho, h\right\},
$$

and taking the perturbation datum $\delta=\left(\downarrow\left(-d_{s}\right) \uparrow\right)^{[]}$, the contraction $c_{A}$ is obtained by applying the Basic Perturbation Lemma. This technique will allow us to extend this result to the case of $A$ being an $A_{\infty}$-algebra, obtaining a contraction between the reduced cobar construction of the tilde bar construction of $A, \bar{\Omega} \widetilde{B} A$, and $A$.

Now we expose the main theorem of the paper. Afterwards, we will dualize the result for $A_{\infty}$-coalgebras.

Theorem 4.1. Let $M$ be a $D G$-module endowed with an $A_{\infty}$-algebra structure. Then there exists a contraction, $c:\left\{A_{M}, M, f, g, \phi\right\}$, between a connected $D G$-algebra $A_{M}$ and the $D G$-module $M$, such that the application of the tensor trick to $c$ provides the original $A_{\infty}$-algebra structure on $M$. 
Proof.

Let $\left(M, m_{1}, m_{2}, \ldots\right)$ be an $A_{\infty}$-algebra and let $\left\{\bar{\Omega} T^{c}(s \bar{M}), M, \alpha, \rho, h\right\}$ be the contraction whose component morphisms are described ahead. In order to make clearer the formulas, we denote by $c_{i}$ the $i$-th component an element of the cobar, $\left\langle c_{1}|\cdots| c_{n}\right\rangle$, with length $k(i) \geq 1$, that is, $c_{i}=\left[a_{i, 1}|\cdots| a_{i, k(i)}\right]$ and we will only specify the whole expression $\left[a_{i, 1}|\cdots| a_{i, k(i)}\right]$ in the case we want emphasize its composition. Particularly, when $k(i)=1$, we write $\left[a_{i}\right]$.

- $\alpha: \bar{\Omega} T^{c}(s \bar{M}) \rightarrow M$,

$$
\begin{aligned}
& \alpha\left\langle c_{1}|\cdots| c_{n}\right\rangle=0 \text { if } n>1 \text { or } k(1)>1 ; \\
& \alpha\langle[a]\rangle=a ;
\end{aligned}
$$

- $\rho: M \rightarrow \bar{\Omega} T^{c}(s \bar{M})$,

$$
\rho(a)=\langle[a]\rangle
$$

- $h: \bar{\Omega} T^{c}(s \bar{M}) \rightarrow \bar{\Omega} T^{c}(s \bar{M})$

$$
\begin{aligned}
& h\left\langle c_{1}|\cdots| c_{n}\right\rangle=0 \text { if } n=0 \text { or } n=1 \text { or } k(1)>1 \\
& \left.\left.h\left\langle\left[a_{1}\right]\left|c_{2}\right| \cdots\right| c_{n}\right]\right\rangle=(-1)^{\left|a_{1}\right|+1}\left\langle\left[a_{1}\left|a_{2,1}\right| \cdots \mid a_{2, k(2)}\right]\left|c_{3}\right| \cdots \mid c_{n}\right\rangle
\end{aligned}
$$

Now the perturbation $\delta$ will consist in including $\left(m_{2}, m_{3}, \ldots\right)$ in the tensor differential of the cobar, so that $\left(\bar{\Omega} T^{c}(s \bar{M}), d_{\bar{\Omega}}+\delta\right)$, becomes $\bar{\Omega} \widetilde{B}(M)$ :

$$
\delta=\left(\downarrow\left(-\sum_{k \geq 2}\left(\uparrow m_{k} \downarrow^{\otimes k}\right)^{[]}\right) \uparrow\right)^{[]} .
$$

The composition $h \delta$ is pointwise nilpotent, since $\delta$ decreases the simplicial degree of the components on the bar construction and $h$ decreases the number of components on the cobar.

Then, we apply the Basic Perturbation Lemma, obtaining the contraction

$$
c_{M}:\{\bar{\Omega} \widetilde{B}(M), M, f, g, \phi\} .
$$

Notice that for an element $\omega=\left\langle\left[a_{1}\right]\left|c_{2}\right| \cdots \mid c_{n}\right\rangle$

$$
\begin{gathered}
\delta h(\omega)=(-1)^{\left|a_{1}\right|+\zeta_{k(2)+1}}\left\langle\left[m_{k(2)+1}\left(a_{1} \otimes a_{2,1} \otimes \cdots \otimes a_{2, k(2)}\right)\right]\left|c_{3}\right| \cdots \mid c_{n}\right\rangle \\
+ \text { other summands with } k(1)>1,
\end{gathered}
$$


where $\zeta_{k(2)+1}=k(2)\left|a_{1}\right|+(k(2)-1)\left|a_{2,1}\right|+\cdots+\left|a_{2, k(2)-1}\right|+[(k(2)+1) / 2]$.

Then, we can describe recursively $(\delta h)^{i}$, up to sign:

$$
\begin{gathered}
(\delta h)^{i}(\omega)=\left\langle\left[m_{k(i+1)+1}\left(\pi_{1,1}\left((\delta h)^{i-1} \omega\right) \otimes a_{i+1,1} \otimes \cdots \otimes a_{i+1, k(i+1)}\right)\right]\right. \\
\left|c_{i+2}\right| \cdots\left|c_{n}\right\rangle
\end{gathered}
$$

where $\pi_{1,1}\left\langle\left[a_{1,1}|\cdots| a_{1, k(1)}\right]\left|c_{2}\right| \cdots \mid c_{n}\right\rangle=a_{1,1}$.

Taking into account these notes, we can describe the morphisms of the contraction above:

- $f(\omega)= \pm m_{k(n)+1}\left(\pi_{1,1}\left((\delta h)^{n-2} \omega\right) \otimes a_{n, 1} \otimes \cdots \otimes a_{n, k(n)}\right)$.

In the particular case of the element $\left\langle\left[a_{1}\right] \mid c_{2}\right\rangle$,

$$
f\left\langle\left[a_{1}\right] \mid c_{2}\right\rangle=(-1)^{1+\left|a_{1}\right|+\zeta_{k(2)+1}} m_{k(2)+1}\left(a_{1} \otimes a_{2,1} \otimes \cdots \otimes a_{2, k(2)}\right) .
$$

- $g=\rho$, since $\delta\langle[a]\rangle=0$.

- $\phi(\omega)=\sum_{i=1}^{n-1}(-1)^{i-1} h(\delta h)^{i-1}\left\langle\left[a_{1}\right]\left|c_{2}\right| \cdots \mid c_{n}\right\rangle$

$$
\begin{gathered}
=(-1)^{\left|a_{1}\right|+1}\left\langle\left[a_{1}\left|a_{2,1}\right| \cdots \mid a_{2, k(2)}\right]\left|c_{3}\right| \cdots \mid c_{n}\right\rangle \\
+\sum_{i=2}^{n-1} \pm\left\langle\left[m_{k(i)+1}\left(\pi_{11}\left((\delta h)^{i-2} \omega\right) \otimes a_{i, 1} \otimes \cdots a_{i, k(i)}\right)\left|a_{i+1,1}\right| \cdots \mid a_{i+1, k(i+1)}\right]\right. \\
\left|c_{i+2}\right| \cdots\left|c_{n}\right\rangle
\end{gathered}
$$

Particularly, for an element $\left\langle\left[a_{1}\right] \mid c_{2}\right\rangle$,

$$
\phi\left\langle\left[a_{1}\right] \mid c_{2}\right\rangle=(-1)^{\left|a_{1}\right|+1}\left\langle\left[a_{1}\left|a_{2,1}\right| \cdots \mid a_{2, k(2)}\right]\right\rangle .
$$

Besides, the perturbed differential on $M$ is zero since $\delta \rho=0$, what means that $M$ still remains with the same differential structure.

We can check now that the structure of $A_{\infty}$-algebra generated on $M$ via the tensor trick coincides with the original infinite structure on $M$.

Take the contraction $T\left(s c_{M}\right):\{T(s \overline{\bar{\Omega} \widetilde{B}(M)}), T(s \bar{M}), T f, T g, T(-\phi)\}$ and the simplicial differential, $d_{s}$, that depends on the juxtaposing product $\mu_{\bar{\Omega}}$, as a perturbation datum. The pointwise nilpotency of $T(-\phi) d_{s}$ is due to the fact that $d_{s}$ does not modify the sum of the lengths of the components of the cobar, meanwhile $T(-\phi)$ decreases, at least by one, this amount. Then, applying the Basic Perturbation Lemma, the following contraction is obtained

$$
\left(f_{s}, g_{s}, \phi_{s}\right): \bar{B} \bar{\Omega} \widetilde{B}(M) \Rightarrow(T(s \bar{M}), \widetilde{d}) .
$$

In order to shorten the formulas ahead, we will denote the iterated composition of morphisms $\phi^{[\otimes i]} \mu^{(i)} \cdots \phi^{\left[{ }^{[\otimes]}\right]} \mu^{(j)}$, with $i \leq j$, by $\Psi^{(i, j)}$ and $\phi^{[\otimes i]} \mu^{(i)}$, simply by $\Psi^{(i)}$. So, the family of 
morphisms given in section 3 that provides an $A_{\infty}$-algebra structure for $M$ can be expressed as follows:

$$
\widetilde{m}_{n}=(-1)^{n+1} f \mu^{(1)} \Psi^{(2, n-1)} g^{\otimes n} .
$$

We will prove that each morphism $\widetilde{m}_{n}$ is exactly the original $m_{n}$ of the $A_{\infty}$-algebra structure on $M$. Take $a=a_{1} \otimes \cdots \otimes a_{n} \in M^{\otimes n}$. Then,

$$
\begin{gathered}
\mu^{(n-1)} g^{\otimes n}(a)=\sum_{i=0}^{n-2}(-1)^{i+1}\left\langle\left[a_{1}\right]\right\rangle \otimes \cdots \otimes\left\langle\left[a_{i}\right]\right\rangle \otimes\left\langle\left[a_{i+1}\right] \mid\left[a_{i+2}\right]\right\rangle \\
\otimes\left\langle\left[a_{i+3}\right]\right\rangle \otimes \cdots \otimes\left\langle\left[a_{n}\right]\right\rangle .
\end{gathered}
$$

The only non-null summand of $\phi^{[\otimes n-1]}$ that can be now applied on each summand on the right hand side is $1^{\otimes i} \otimes \phi \otimes(g f)^{\otimes n-i-2}$, where $\phi$ is applied (following (4)) to the only element of cosimplicial dimension 2 .

$$
\begin{gathered}
\phi^{[\otimes n-1]} \mu^{(n-1)} g^{\otimes n}(a)=\sum_{i=0}^{n-2}(-1)^{i+1+\gamma_{i}}\left\langle\left[a_{1}\right]\right\rangle \otimes \cdots \otimes\left\langle\left[a_{i}\right]\right\rangle \otimes\left\langle\left[a_{i+1} \mid a_{i+2}\right]\right\rangle \\
\otimes\left\langle\left[a_{i+3}\right]\right\rangle \otimes \cdots \otimes\left\langle\left[a_{n}\right]\right\rangle,
\end{gathered}
$$

where $\gamma_{i}=\left|a_{1}\right|+\cdots\left|a_{i}\right|+\left|a_{i+1}\right|+1$.

Notice that the obtained summands have again, only factors of cosimplicial dimension 1 and this condition remains every time that $\Psi^{(k)}$, for any $k$, is applied: take an element from $(\bar{\Omega} \widetilde{B}(M))^{\otimes j}$ in the form $\bar{\omega}=\left\langle c_{1}\right\rangle \otimes \cdots \otimes\left\langle c_{j}\right\rangle$, then,

$$
\mu^{(j-1)}(\bar{\omega})=\sum_{i=0}^{j-2}(-1)^{i+1}\left\langle c_{1}\right\rangle \otimes \cdots \otimes\left\langle c_{i}\right\rangle \otimes\left\langle c_{i+1} \mid c_{i+2}\right\rangle \otimes\left\langle c_{i+3}\right\rangle \otimes \cdots \otimes\left\langle c_{j}\right\rangle,
$$

so $\phi^{[\otimes j-1]}$ (on each summand above) is reduced to $1^{\otimes i} \otimes \phi \otimes(g f)^{\otimes j-i-2}$ where $\phi$ is applied to the only factor of cosimplicial degree 2 and will be non-null only if $c_{i+1}=\left[a_{i+1}\right]$,

$$
\begin{gathered}
\Psi^{(j-1)}(\bar{\omega})=\sum_{i=0}^{j-2}(-1)^{i+1+\gamma_{i}}\left\langle c_{1}\right\rangle \otimes \cdots \otimes\left\langle c_{i}\right\rangle \otimes\left\langle\left[a_{i+1}\left|a_{i+2,1}\right| \cdots \mid a_{i+2, k(i+2)}\right]\right\rangle \\
\otimes g f\left\langle c_{i+3}\right\rangle \otimes \cdots \otimes g f\left\langle c_{j}\right\rangle,
\end{gathered}
$$

with $\gamma_{i}=\left|c_{1}\right|+\cdots+\left|c_{i}\right|+i+\left|a_{i+1}\right|+1$.

Besides, the elements $f\left\langle c_{i+3}\right\rangle, \ldots f\left\langle c_{j}\right\rangle$ will be non-null only if $k(i+3)=1=\cdots=k(j)$. In this case, the $i$-th summand of (5) will be

$$
\left\langle c_{1}\right\rangle \otimes \cdots \otimes\left\langle c_{i}\right\rangle \otimes\left\langle\left[a_{i+1}\left|a_{i+2,1}\right| \cdots \mid a_{i+2, k(i+2)}\right]\right\rangle \otimes\left\langle\left[a_{i+3}\right]\right\rangle \otimes \cdots \otimes\left\langle\left[a_{j}\right]\right\rangle,
$$

which has only factors with 1 component. 
This way, in order to obtain a non-null result for the $i$-th summand, the element to which $\Psi^{(j-1)}$ is applied must be

$$
\left\langle c_{1}\right\rangle \otimes \cdots \otimes\left\langle c_{i}\right\rangle \otimes\left\langle\left[a_{i+1}\right]\right\rangle \otimes\left\langle c_{i+2}\right\rangle \otimes\left\langle\left[a_{i+3}\right]\right\rangle \otimes \cdots \otimes\left\langle\left[a_{j}\right]\right\rangle .
$$

As for the application of the whole sequence $\mu^{(1)} \Psi^{(2, n-1)}$, starting from a tensor product of $n$ elements from the cobar construction, the morphism $\mu^{(i)}$ always decreases by one the number of factors from the cobar construction and $\phi^{[\otimes i]}$ does not touch this amount. This way, after applying $\mu^{(1)} \Psi^{(2, n-1)}$, only one factor is obtained to which $f$ will be applied. Taking this into account together with the fact that the only elements that survive to $\Psi^{(j-1)}$ are those in the form (6), we conclude that the only summand of $\Psi^{(j-1)}$ that will pass trough $f \mu^{(1)} \Psi^{(2, j-2)}$ is that of $i=j-2$ in the sum (5), whenever $c_{j-1}=\left[a_{j-1}\right]$. Then,

$$
\Psi^{(j-1)}(\bar{\omega})=(-1)^{j-1+\gamma_{j-2}}\left\langle c_{1}\right\rangle \otimes \cdots \otimes\left\langle c_{j-2}\right\rangle \otimes\left\langle\left[a_{j-1}\left|a_{j, 1}\right| \cdots \mid a_{j, k(j)}\right]\right\rangle+\cdots,
$$

where dots represent the rest of summands that, from now on, we will omit.

Now, by induction on $k$, one can easily prove that

$$
\Psi^{(n-k, n-1)} g^{\otimes n}(a)=(-1)^{\varrho(k)}\left\langle\left[a_{1}\right]\right\rangle \otimes \cdots \otimes\left\langle\left[a_{n-k-1}\right]\right\rangle \otimes\left\langle\left[a_{n-k}|\cdots| a_{n}\right]\right\rangle,
$$

where $\varrho(k)=\sum_{i=0}^{k-1}(n-i)+\sum_{i=1}^{k} i\left|a_{n-i}\right|+k \sum_{i=k+1}^{n-1}\left|a_{n-i}\right|$.

So, for the case $k=n-2$,

$$
\Psi^{(2, n-1)} g^{\otimes n}(a)=(-1)^{\varrho(n-2)}\left\langle\left[a_{1}\right]\right\rangle \otimes\left\langle\left[a_{2}|\cdots| a_{n}\right]\right\rangle,
$$

where

$$
\begin{aligned}
\varrho(n-2) & =\sum_{i=0}^{n-3}(n-i)+\sum_{i=1}^{n-2} i\left|a_{n-i}\right|+(n-2)\left|a_{1}\right| \\
& \equiv[(n-1) / 2]+\sum_{i=1}^{n-2} i\left|a_{n-i}\right|+(n-2)\left|a_{1}\right|(\bmod 2) .
\end{aligned}
$$

Taking up again the calculation of $\widetilde{m}_{n}$,

$$
\begin{aligned}
\widetilde{m}_{n}\left(a_{1} \otimes \cdots \otimes a_{n}\right) & =(-1)^{n+1+\varrho(n-2)} f \mu^{(1)}\left\langle\left[a_{1}\right]\right\rangle \otimes\left\langle\left[a_{2}|\cdots| a_{n}\right]\right\rangle \\
& =(-1)^{n+\varrho(n-2)} f\left\langle\left[a_{1}\right] \mid\left[a_{2}|\cdots| a_{n}\right]\right\rangle \\
(\text { using 3) } & =(-1)^{n+\varrho(n-2)+1+\left|a_{1}\right|+\zeta_{n}} m_{n}\left(a_{1} \otimes \cdots \otimes a_{n}\right),
\end{aligned}
$$

where $\zeta_{n}=\sum_{i=1}^{n-1} i\left|a_{n-i}\right|+[n / 2]$ and hence

$$
n+\varrho(n-2)+1+\left|a_{1}\right|+\zeta_{n} \equiv 0(\bmod 2) .
$$


That is, the $A_{\infty}$-algebra structure obtained coincides with the original one defined on $M$.

This theorem provides a structural representation of an $A_{\infty}$-algebra as a contraction.

Corolary 4.2. A DG-module $M$ is endowed with an $A_{\infty}$-algebra structure if and only if there exists a contraction $c:\left\{A_{M}, M, f, g, \phi\right\}$, between a connected $D G$-algebra $A_{M}$ and a $D G$-module $M$.

Now, we show the dual result to the theorem given above, in the case of $A_{\infty}$-coalgebras, omitting its proof since it follows a similar scheme (though quite more tedious). From now on, all the DG-modules will be considered to be simply connected. This way, any $A_{\infty}$-coalgebra can be represented as a contraction from a simply connected DG-coalgebra.

Theorem 4.3. Let $M$ be a $D G$-module endowed with an $A_{\infty}$-coalgebra structure. Then there exists a contraction $c:\left\{C_{M}, M, f, g, \phi\right\}$, between a simply connected $D G$-coalgebra $C_{M}$ and the DG-module $M$, such that the application of the tensor trick to $c$ yields the initial $A_{\infty}$-coalgebra structure on $M$.

In this case, the simply connection guarantees that the perturbation process is finite and a contraction $c_{M}:\{\bar{B} \widetilde{\Omega}(M), M, f, g, \phi\}$ can be constructed.

Corolary 4.4. A $D G$-module $M$ is endowed with an $A_{\infty}$-coalgebra structure if and only if there exists a contraction $c:\left\{C_{M}, M, f, g, \phi\right\}$, between a simply connected $D G$-coalgebra $C_{M}$ and a $D G$-module $M$.

We are aware that, at this point, the development of a categorical framework for $A_{\infty}-$ (co)algebras in terms of chain contractions would be a natural direction of our future efforts.

\section{References}

[BV73] J.M. Boardman, R.M. Vogt (1973), Homotopy invariant algebraic structures on topological spaces. Lecture Notes in Mathematics, Springer-Verlag, 347.

[Bro67] R. Brown (1967), The twisted Eilenberg-Zilber theorem, Celebrazioni Archimedee del secolo XX, Simposio di topologia, 34-37.

[CE56] H. Cartan (1956), S. Eilenberg. Homological Algebra. Princeton University Press, Princeton.

[EM53] S. Eilenberg, S. Mac Lane (1953), On the groups $H(\pi, n)-\mathrm{I}$, Annals of Math. 58 $55-106$.

[Gug72] V.K.A.M. Gugenheim (1972), On the chain complex of a fibration, Illinois J. Math. $3398-414$. 
[Gug77] V.K.A.M. Gugenheim (1977), On Chen's iterated integrals, Illinois J. Math. 703715 .

[GL89] V.K.A.M. Gugenheim, L.A. Lambe(1989), Perturbation theory in Differential Homological Algebra I, Illinois J. Math. 33 (4) 566-582.

[GLS91] V.K.A.M. Gugenheim, L.A. Lambe, J.D. Stasheff (1991), Perturbation Theory in Differential Homological Algebra II, Illinois J. Math. 35 (3) 357-373.

[GS86] V. K. A. M. Gugenheim and J. Stasheff (1986), On Perturbations and $A_{\infty^{-}}$ structures, Bull. Soc. Math. Belg. 38 237-246.

[HK91] J. Huebschmann, T. Kadeishvili (1991), Small models for chain algebras, Math. Z. $207245-280$.

[Kad80] T. Kadeishvili (1980), On the Homology Theory of Fibrations, Russian Math. Surveys 35 (3) 231-238.

[LS87] L.A. Lambe, J.D. Stasheff (1987), Applications of perturbation theory to iterated fibrations, Manuscripta Math. 58 367-376.

[Mac95] S. Mac Lane (1995), Homology, Classics in Mathematics Springer-Verlag, Berlin. Reprint of the 1975 edition.

[Mun74] H.J. Munkholm (1974), The Eilenberg-Moore spectral sequence and strongly homotopy multiplicative maps. J. Pure Appl. Alg. 5 1-50.

[Pro84] A. Prouté (1984), Algebrès diffeérentielles fortement homotopiquement associatives ( $A_{\infty}$-algèbre), Ph. D. Thesis, Université Paris VII.

[Rea00] P. Real (2000), Homological Perturbation Theory and Associativity, J. Homology, Homotopy and Appl. 2 51-88.

[Shi62] W. Shih (1962), Homologie des espaces fibré, Inst. Hautes Etudes Sci. 13 293-312.

[Sta63] J.D. Stasheff (1963), Homotopy Associativity of $H$-spaces I, II, Trans. A.M.S, 108 $275-312$.

[SU04] S. Saneblidze, R. Umble (2004), Diagonals on the permutahedra, multiplihedra and associahedra, J. Homology, Homotopy and Applications, 6 (1) 363-411. 